\title{
Extracting clean supernova spectra ${ }^{\star}$
}

\section{Towards a quantitative analysis of high-redshift Type la supernova spectra}

\author{
S. Blondin ${ }^{1}$, J. R. Walsh ${ }^{2}$, B. Leibundgut ${ }^{1}$, and G. Sainton ${ }^{3}$ \\ 1 European Southern Observatory (ESO), Karl-Schwarzschild-Strasse 2, 85748 Garching bei München, Germany \\ e-mail: [sblondin;bleibund] @eso.org \\ 2 Space Telescope-European Coordinating Facility (ST-ECF), European Southern Observatory (ESO), \\ Karl-Schwarzschild-Strasse 2, 85748 Garching bei München, Germany \\ e-mail: jwalsh@eso.org \\ 3 LPNHE CNRS/IN2P3, Université Paris VI \& VII, 4 place Jussieu, 75252 Paris Cedex 05, France \\ e-mail: sainton@in2p3.fr
}

Received 14 September 2004 / Accepted 14 October 2004

\begin{abstract}
We use a new technique to extract the spectrum of a supernova from that of the contaminating background of its host galaxy, and apply it to the specific case of high-redshift Type Ia supernova (SN Ia) spectroscopy. The algorithm is based on a two-channel iterative technique employing the Richardson-Lucy restoration method and is implemented in the IRAF code specinholucy. We run the code both on simulated (SN Ia at $z=0.5$ embedded in a bright host galaxy) and observed (SNe Ia at various phases up to $z=0.236$ ) data taken with VLT+FORS1 and show the advantages of using such a deconvolution technique in comparison with less elaborate methods. This paper is motivated by the need for optimal supernova spectroscopic data reduction in order to make meaningful comparisons between the low and high-redshift SN Ia samples. This may reveal subtle evolutionary and systematic effects that could depend on redshift, and bias the cosmological results derived from comparisons of local and high- $z$ SNe Ia in recent years. We describe the various aspects of the extraction in some detail as guidelines for the first-time user and present an optimal observing strategy for successful implementation of this method in future high- $z \mathrm{SN}$ Ia spectroscopic follow-up programmes.
\end{abstract}

Key words. stars: supernovae: general - stars: supernovae: individual: SN 2002bo, SN 2002go, SN 2002gr instrumentation: spectrographs - methods: data analysis - techniques: spectroscopic

\section{Introduction}

The initial claim made by two independent teams - the High-Z Supernova Search Team (Schmidt et al. 1998) and the Supernova Cosmology Project (Perlmutter et al. 1999) - that the apparent dimming of Type Ia supernovae (SN Ia) at redshifts of $z \approx 0.5$ implies a present accelerating expansion of the universe (Riess et al. 1998a; Perlmutter et al. 1999), and its subsequent confirmation with improved precision (Tonry et al. 2003; Knop et al. 2003; Barris et al. 2004), have prompted an increased interest in these astrophysical events. On the theoretical side, much effort and computing time has been devoted to multi-dimensional modelling of the explosion in order to provide physical input parameters for spectral synthesis calculations (see Hillebrandt \& Niemeyer 2000, for a review). On the observational side several teams are currently using SNe Ia both to probe the decelerating expansion at higher $(z \gtrsim 1)$ redshifts - the Hubble Higher- $z$ Supernova Search

^ Partly based on observations collected at the European Southern Observatory, Chile (ESO Programme 170.A-0519).
(Riess et al. 2004) - and to measure second order effects (the equation-of-state parameter $\omega=p / \rho c^{2}$ ) at intermediate $(0.2 \lesssim z \lesssim 0.8)$ redshifts - such as ESSENCE (Miknaitis et al. 2005; Matheson et al. 2005) and the CFHT SuperNova Legacy Survey, or SNLS (Pain \& SNLS Collaboration 2002).

However, the physical parameters governing the SN Ia explosion mechanism are today not fully understood and the empirical parametrisation of the entire SN Ia class - the so-called "Phillips relation" (see Phillips 1993) - has no physical basis. The aforementioned observational programmes will detect several hundreds of high- $z \mathrm{SNe}$ Ia, at which stage the statistical errors in the sample will have reached the systematic error floor. The cosmological effect detected via observations of Type Ia supernovae is indeed a subtle one (see Leibundgut 2001, for a review) and the field is limited by the systematic errors involved in such measurements and their possible evolution with redshift.

Spectroscopy is an ideal way to probe these potential evolutionary effects through systematic comparison of high$z$ SN Ia spectra with local templates. This requires the 
extraction of clean SN Ia spectra devoid of background contamination, mainly by the host galaxy and sky lines (for groundbased observations). For high signal-to-noise (hereafter $\mathrm{S} / \mathrm{N}$ ) cases, when the supernova is bright with respect to its immediate underlying background, standard extraction software will in general work well. However, when the supernova is faint compared to the background, e.g. at late phases, heavily superposed on its host galaxy or simply at a high redshift, a more elaborate technique is required to ensure that we are not extracting nonSN flux.

The purpose of this paper is to test such a method that has been implemented in the $\operatorname{IRAF}^{1}$ code specinholucy (Lucy \& Walsh 2003). It is based on a two-channel restoration algorithm that restores a point spread function (PSF)-like component in a 2D image and an underlying extended background separately. It is of wide astronomical use and has already been successfully applied to the restoration of point-source spectra in highly inhomogeneous backgrounds (see Lucy \& Walsh 2003), such as is often the case for high- $z$ SNe Ia embedded in their host galaxy. The fact that the entire 2D spectrum of the background (with or without the inclusion of the restored point source spectrum) is restored in such an algorithm means that we have a firm hold on potential systematic errors associated with the restoration. This is done by simply comparing the residuals in the restored $2 \mathrm{D}$ spectrum with the statistical noise limit of the input 2D spectrum (the square root of the number of photoelectrons). Should these residuals fall below this limit then we can consider the restoration to be successful. This is a clear advantage over traditional spectral extraction routines where no secure hold on systematic errors is possible. Furthermore, the very nature of the algorithm presented in this paper implies that all non PSF-like components in the input 2D spectrum are automatically allocated to the background channel. We are making no $a$ priori assumptions on the nature of the contaminating extended background component and only rely on the point-like nature of the supernova's spatial profile.

In Sect. 2 we present the algorithm and its application to the case of supernovae embedded in their host galaxy. We dedicate Sects. 3 and 4 to the specific issues of the PSF spectrum - or slit spread function (SSF) - used to restore the point source and of the spatial resolution kernel used to discriminate between the point source and the underlying extended background, respectively, as they are of crucial importance to the proper functioning of specinholucy. In Sects. 5 and 6 we test the algorithm on simulated and observed data, respectively. Section 7 serves the purpose of comparing our technique to alternative spectral extraction methods, and we further discuss the advantages of using our approach alongside an optimal observing strategy for its successful implementation in Sect. 8.

The need for comparisons between the high- $z$ and the local SN Ia spectra in order to make quantitative statements on their potential differences requires the use of such an elaborate two-channel deconvolution technique. The code specinholucy

${ }^{1}$ IRAF is distributed by the National Optical Astronomy Observatories, operated by the Association of Universities for Research in Astronomy, Inc., under contract to the National Science Foundation of the United States. enables a truly quantitative analysis of high- $z$ SN Ia spectra and opens the path for meaningful detections of potential evolutionary signatures in their spectra.

\section{The algorithm and its implementation}

\subsection{Decomposing the $S N$ from its host galaxy}

Lucy \& Walsh (2003) - subsequently LW03 - have described two iterative techniques for decomposing a long-slit spectrum into spectra of designated point sources and an underlying background. The methods are based on a two-channel restoration (Hook \& Lucy 1994; Lucy 1994) using the RichardsonLucy (Richardson 1972; Lucy 1974) iterative restoration. The essence of this technique is to treat the point source(s) and the extended background as two channels for restoration. It must be emphasized at the outset that this restoration occurs purely in the spatial direction and no implied spectral restoration is undertaken. Thus, for instance, residuals from fringing corrections should not be relevant unless the fringes are tilted with respect to the dispersion axis, in which case an additional inhomogeneous component would be added to the background. The first channel contains the point source(s) which are restored to delta functions using an appropriate PSF. For a spectrum the PSF must be specified as a function of wavelength; this is simply the aforementioned SSF in Lucy \& Walsh (2003). In order to iteratively restore the extended component, it is necessary to impose a limiting resolution, larger than that set by the PSF, in order to prevent the second channel from modelling the point sources as peaks in the extended component. The resolution limit for the background is set by defining the restored background to be the convolution of a non-negative auxiliary function with a wavelength-independent resolution kernel $R$. Then no feature whose width is less than $R$ can appear in the restored background. If the width of $R$ is greater than the PSF, then the convolution of the model of the extended background $\psi$ with the PSF cannot fit a point source, which must therefore be modelled by the first channel.

Two methods are described in LW03: one for a homogeneous background (i.e. the spectrum of the background is homogeneous - does not vary with position); the second, more general, case allows the spectrum of the background to vary as a function of position along the slit - termed the inhomogeneous background case. In the case of a galaxy including an $\mathrm{SN}$ the homogeneous case is appropriate. The observed spectrum $\phi(\lambda, y)$, where $\lambda$ is the dispersion direction and $y$ the crossdispersion direction, can then be modelled as:

$\phi(\lambda, y)=\int F(\lambda, \eta) P(\lambda, y-\eta) \mathrm{d} \eta+f_{\mathrm{SN}}(\lambda) P\left(\lambda, y-y_{\mathrm{SN}}\right)$,

where $\eta$ is the independent variable for the spatial dimension (cross-dispersion direction). The first term represents the spectrum of the background and the second represents the spectrum of the SN. In this study it is $f_{\mathrm{SN}}(\lambda)$, the spectrum of the $\mathrm{SN}$, which is the desired output product. $P(\lambda, y-\eta)$ is the SSF, i.e. a PSF as a function of wavelength. The restored background, $\psi$, is a convolution of an non-negative auxilliary function $\chi(\lambda, \zeta)$ 
and the non-negative normalized resolution kernel centred at $\eta=\zeta, R(\eta-\zeta)$ :

$F(\lambda, \eta)=\int \chi(\lambda, \zeta) R(\eta-\zeta) \mathrm{d} \zeta$,

which is convolved with the SSF to match the observed extended component. $R$ is specified as a Gaussian function of sigma $\sigma_{\text {kernel }}$.

The unknowns $f_{\mathrm{SN}}$ and $\chi$ are determined by iterative improvement of the fit of $\phi$ to the observed spectrum, using the $R-L$ algorithm to improve both the point source spectrum and the background until convergence is reached. The iteration steps are described in detail in LW03. There are two convergence criteria: the fractional change in the spectrum per $R-L$ iteration summed for all wavelengths; the fractional change in $\chi$ per iteration summed over all wavelengths and cross-dispersion range. In practice, depending on the particular details of the spectrum, one of these criteria may converge much faster than the other, though both must be met for the code to converge.

\subsection{Practical implementation for SN and host galaxy}

The situation of an SN observed in a host galaxy is an ideal use of the inhomogeneous case (see LW03, for some other examples). This is the simplest application since there is ideally one point source and a structurally well-resolved galaxy. When the $\mathrm{SN}$ is in the outer regions of an early-type galaxy, then the sophistication of the technique is probably not essential and more traditional methods, such as a linear or polynomial fit to the background and extraction, with or without weighting, of the point source, are adequate. However, when the $\mathrm{SN}$ is near the centre of a galaxy, or the galaxy does not have a smooth radial profile, or there is line emission in the galaxy host (e.g. for late-type galaxies), then the simple methods fail and a dedicated extraction or a restoration approach is mandatory. In Sect. 7 we compare the technique presented here with other less advanced methods.

The inhomogeneous case is implemented in the IRAF code specinholucy, which is available in the ST-ECF layered package specres. In addition to the input long-slit 2D spectrum, there are two important input parameters: the SSF, which obviously must match as well as possible the cross-dispersion profile as a function of wavelength for the SN observation, and the position of the SN on the slit. The inhomogeneous decomposition technique needs the position of the point source that is to be extracted from the extended background (i.e. the host galaxy). Errors in this position will result in mixing of the two channels in the output-restored spectrum so that for example the background is excavated asymmetrically around the point source. However, this requirement is not as limiting as it sounds since one of the outputs is a $2 \mathrm{D}$ restored version of the input $2 \mathrm{D}$ long-slit spectrum (including both the $\mathrm{SN}$ and the host galaxy) which can be directly compared with the latter spectrum, and a mismatch of the position shows up as a higher frequency component than the resolution kernel in the neighbourhood of the designated SN. The primary output is the spectrum of the SN, which is strictly 1D. If an error image is available for the input long-slit spectrum, it can be supplied to produce Monte Carlo error estimates for the restored output spectra (point source and extended background).

\section{The importance of the slit spread function}

LW03 discuss some of the difficulties with using an empirical SSF. For ground-based data a spectrum of a bright standard star is ideal, but this must be taken in identical conditions to those of the $\mathrm{SN}$ spectrum. In practice this is almost impossible to achieve. Even a star on the slit of the same observation as the SN cannot be guaranteed to provide an ideal SSF, since the star may not be centred on the slit as the $\mathrm{SN}$ is centered and instrumental optical aberrations can produce off-axis distortions of the spatial profile of a point source. A simple task, specps $f$, is provided for constructing model SSFs in the specres package. A number of PSFs (viz stellar images) are provided as input at specified wavelengths; these are sampled by a slit of specified size (relative to the PSFs) and the signal within a slit is integrated across the slit width as a function of offset position (cross-dispersion). The set of point sources are then splineinterpolated in the dispersion direction to provide the 2D spectrum of the point source, the SSF. For an observation with no accompanying SSF, a considerable amount of trial-and-error is required to choose the seeing of a set of model PSFs in order to provide a high quality restoration, and thus successful extraction of the SN spectrum. In the low signal-to-noise cases, it will be the major source of uncertainty in the SN spectrum (see Sect. 5.3). However, all methods that rely on provision of a PSF (in imaging) or an SSF (in spectroscopy) are subject to this difficulty; it usually provides the fundamental limit on the uncertainty of the restoration.

\subsection{Measuring the seeing}

To successfully extract the point source spectrum from the extended background component one needs in principle to know the width of the point-source spatial profile at each dispersion coordinate - i.e. the $F W H M$-wavelength relation for that object. In practice, however, we often cannot do this because of poor $\mathrm{S} / \mathrm{N}$ (this is especially the case in high- $z \mathrm{SN}$ Ia spectroscopy) and so we rely on average quantities, such as the mean seeing during the observation. One can then imagine reconstructing a more reliable description of the seeing $(\theta)$ variation along the dispersion direction $(\lambda)$ using for instance the following seeingwavelength relation (Schroeder 1987):

$\left.\begin{array}{l}\theta=\lambda / r_{0} \\ r_{0} \propto \lambda^{6 / 5}\end{array}\right\} \Rightarrow \theta \propto \lambda^{-0.2}$,

where $r_{0}$ is the so-called Fried parameter, which describes the quality of a wave that has propagated through atmospheric turbulence (Fried 1965, 1975).

However, a specific telescope and instrument combination can introduce systematic errors due to instrumental spatial distortions which are not taken into account in the above relation. Figure 1 shows that one should not rely on measurements by external seeing monitors on observatory sites such as the Differential Image Motion Monitor (DIMM) on 


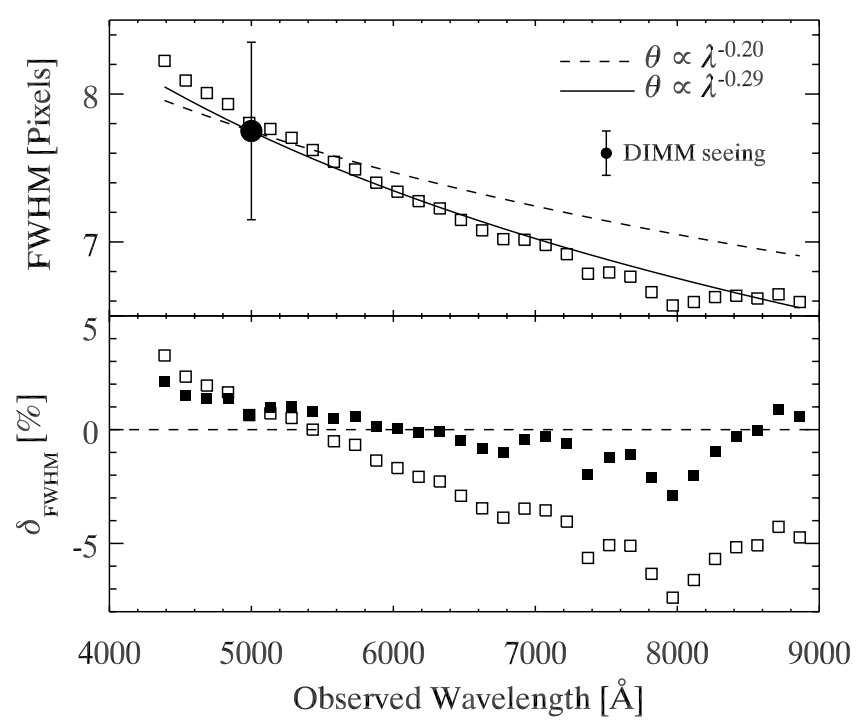

Fig. 1. Top panel: $F W H M$ of the cross-dispersion profile of the standard star LTT 7987 taken with VLT+FORS1 on UT 11 October 2002. The single data point with an error bar shows the mean seeing as measured by the DIMM station - namely $1.55 \pm 0.12^{\prime \prime}$ (the pixel scale of FORS 1 is $0.2^{\prime \prime} \mathrm{pix}^{-1}$ ) at $5000 \AA$. Overplotted is the FWHMwavelength relation fixed at the DIMM data point, both for the predicted exponent $(-0.20)$ and our best-fit value $(-0.29)$. Lower panel: fractional residuals with respect to the $\theta \propto \lambda^{-l}$ relation, for $l=0.20$ (open squares) and $l=0.29$ (filled squares).

the ESO-Paranal site, which measures the image quality via a differential centroiding method (Sarazin \& Roddier 1990; Sandrock et al. 2000). Although precise, these measurements are inadequate for our purpose since the DIMM probes a region of sky significantly different from that where the telescope is pointing (see Patat 2003, Fig. 17). Moreover the optical train is different and the observation wavelength is restricted to $5000 \AA$. Even varying the exponent in the above seeingwavelength relation does not make it possible to accurately reproduce the $F W H M$ profile of a bright standard star (Fig. 1). We will see in Sect. 5.3 that errors of $\gtrsim 15 \%$ in the determination of the FWHM of the point source can lead to significant errors in the restored point source flux. One possible alternative is to construct a synthetic SSF based on the wavelength-dependent FWHM characteristics of the point source spectrum to be extracted. This has the prime advantage of extracting the point source with a PSF spectrum that has been subject to the same seeing variations. To do so one can either use the SN spectrum itself or carefully align the slit to include a bright single star as well as the object of interest.

\subsection{Generating synthetic PSF spectra}

The IRAF-implemented code specpsf is illustrated in Fig. 2. A standard star is used to determine the corresponding FWHMwavelength relation, from which Gaussian PSF images are generated every $50 \AA$. The resulting stellar images are run through specpsf and the profile of the corresponding output PSF spectrum (or SSF) is compared with that of the input standard star. The errors fall below $0.1 \%$ over the whole wavelength range.

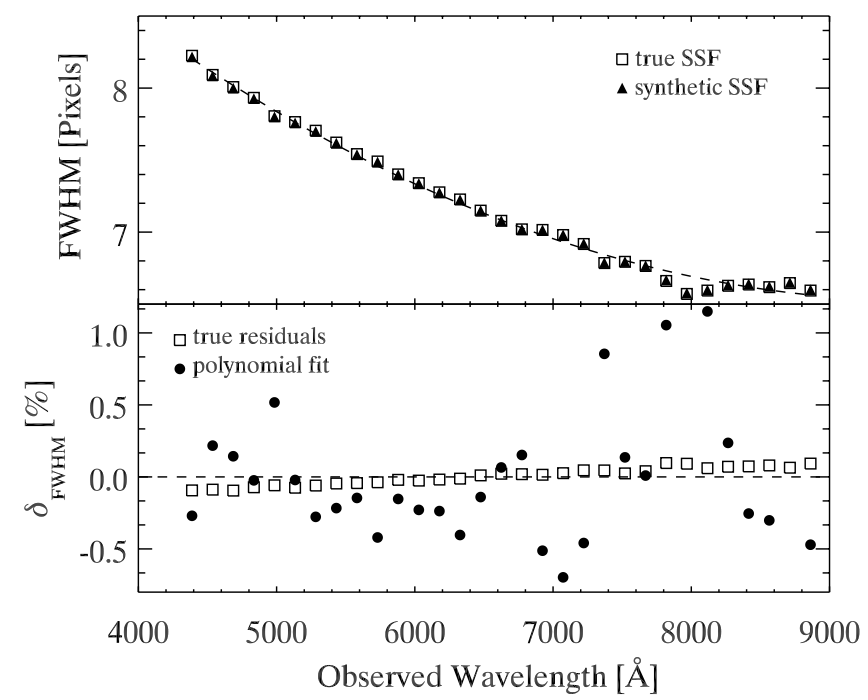

Fig. 2. Illustration of SSF synthesis using specpsf. The input spectrum is the same as in Fig. 1. Top panel: FWHM of LTT 7987 (open squares) and of the synthetic SSF (filled triangles). The dashed line shows a polynomial fit to the synthetic SSF. Lower panel: residual plots of the FWHM of the synthesized SSF (open squares) and polynomial fit to the synthetic SSF (filled circles) with respect to the input SSF.

We also show a polynomial fit to the data, since in practice the scatter is significantly larger for low $\mathrm{S} / \mathrm{N}$ point sources, and such a fit to the data is more representative of the actual seeing variations in the dispersion direction. Here the residuals are as high as $\sim 2 \%$ in the red region of the spectrum (mainly due to an increase of sky brightness in this spectral range), still an order of magnitude below the lower threshold where errors on the FWHM start to matter (see below).

\subsection{Impact of the SSF on spectral restoration}

The astronomer wishing to extract point source spectra using specinholucy has various options for the SSF. In low S/N cases like the ones we describe in this paper the choice of the SSF is by far the most limiting factor and will have a severe impact on the quality of the restoration.

We illustrate the impact of the SSF choice on the restoration of SN 2002go, a Type Ia supernova at $z=0.236$ (IAUC 7994) slightly offset from the center of its host galaxy (see also Sect. 6). We consider three options for the SSF: (1) a synthetic SSF based on FWHM measurements of SN 2002go itself; (2) a PSF star that happened by chance to be on the same slit as SN 2002go; and (3) a VLT+FORS1 standard star for which the $F W H M$-wavelength relation was the closest we could find to that of SN 2002go. The resulting cross-dispersion profiles are shown in Fig. 3, and the corresponding mean seeing values are shown in Table 1. Note that the quoted mean values are not representative of the wavelength-dependent nature of the seeing, and one should produce plots such as those shown in Fig. 3 to appreciate the differences between the SSFs. Despite having been observed on the same location of the CCD chip, the SN and the standard star were not observed on the same night and were thus subject to different seeing conditions. The difference is also a function of wavelength, hinting at possible 


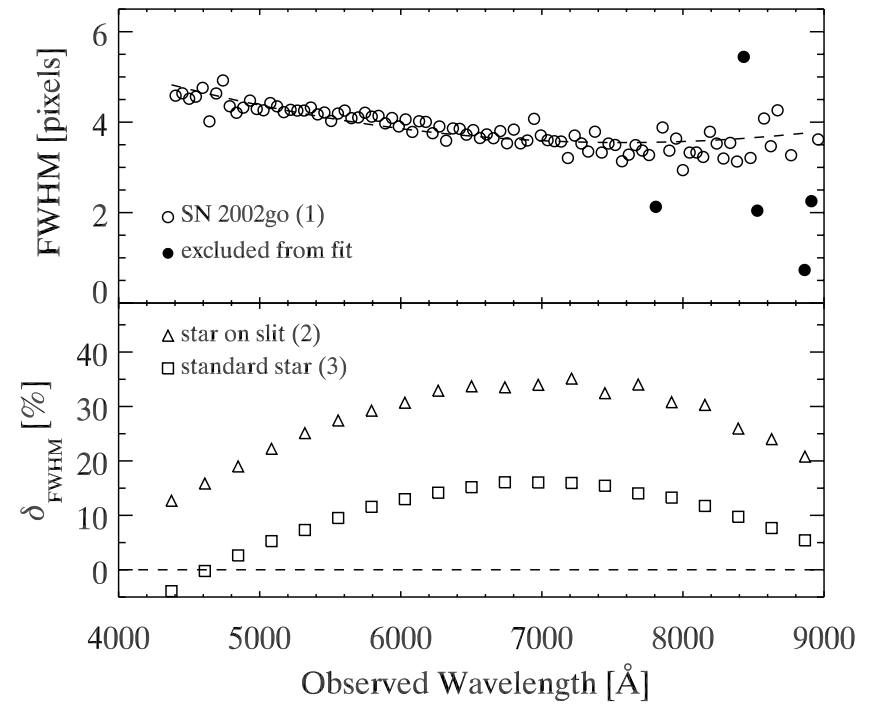

Fig. 3. Examples of SSFs used to extract SN 2002go: (1) the supernova itself; (2) a bright PSF star that happened to be on the same slit; (3) the closest FORS1 standard star to match the supernova profile. Top panel: the dashed line is a polynomial fit to the SN profile, from which low $\mathrm{S} / \mathrm{N}$ points affected by the increase in sky background have been excluded. Lower panel: fractional differences in the SSF FWHM with respect to the polynomial fit.

Table 1. SSF FWHM data.

\begin{tabular}{lll}
\hline \hline SSF & pixels $^{a}$ & arcseconds $^{a}$ \\
\hline SN 2002go & $3.83_{3.48}^{4.74} \pm 0.41$ & $0.77_{0.70}^{0.95} \pm 0.08$ \\
star on slit & $4.71_{4.18}^{5.44} \pm 0.39$ & $0.94_{0.84}^{1.10} \pm 0.08$ \\
standard star & $4.17_{3.93}^{4.64} \pm 0.23$ & $0.83_{0.79}^{0.93} \pm 0.05$ \\
\hline
\end{tabular}

${ }^{a}$ The quoted values are read as $\langle\text { mean }\rangle_{\min }^{\max } \pm 1 \sigma$ error.

instrumental effects on the spatial profile. These same comments apply to standard stars having been observed on the same night as the object of interest, and we discourage the potential user of this method from using standard stars for the SSF.

More interesting, perhaps, are the quasi-systematic differences in the profiles of the SN and the PSF star that was accidentally placed on the same slit. The SN and PSF star should be subject to identical seeing variations, but the reconstructed profiles differ by as much as $\sim 30 \%$, or $0.2^{\prime \prime}$. There could be several reasons for this: (a) the observation is not made at the parallactic angle, and so the dispersion direction is not along the slit; (b) the star spectrum is located at a different location on the CCD chip where the spatial distortion differs; (c) the star is not centred on the slit and we are not measuring its true PSF profile. FORS1 is equipped with a longitudinal atmospheric dispersion compensator (LADC) which has an accuracy $\lesssim 0.1^{\prime \prime}$ ( 0.5 pix) over the considered wavelength range (Avila et al. 1997), and so (a) should not be relevant. FORS1 is linear at the $<0.4 \%$ level (see the FORS1+2 User Manual ${ }^{2}$ ) and distortions associated with the instrument's optics should not affect the whole spectral range at this level, so (b) should not matter either. This leaves (c) as the probable explanation

\footnotetext{
${ }^{2}$ http://www.eso.org/instruments/fors1/
}

for this difference, which is significant enough to be a major source of error in the restoration.

The impact of these various SSFs on the restored SN flux is shown in Fig. 4. We view the solid curve as the most accurate restoration, and one sees that using the other two (wider) SSFs means we are also restoring part of the extended background component (sky and host galaxy) in the point source spectrum. In the case of the star that was by chance located on the same slit the $\sim 20 \%$ difference in the $F W H M$ results in an error in the restored $\mathrm{SN}$ flux that can be as high as $100-150 \%$ ! We see from the two inlays in Fig. 4 that the slope of the pseudocontinuum around strong SN Ia spectral features (Ca II H\&K and $\mathrm{Si}$ II) changes significantly due to contamination of the underlying background spectrum, and so the very definition of "line strength" in this case is very ambiguous. The fact that the $F W H M$ of the point source varies with wavelength will cause variable contributions of the background to the restored spectrum, and will affect the determination of line ratios of distantly spaced lines, if the PSF vector is not determined properly. Thus, the extraction has a significant impact on empirical correlations based on such measurements, as the errors made at this stage will add to those associated with the subsequent calibration in flux. Examples of such correlations are given in Nugent et al. (1995) and are used to determine distances to SNe Ia in Riess et al. (1998b).

One might ask how one can decide which SSF to choose to restore a particular point source spectrum and if it is possible to a posteriori tell whether the chosen SSF was indeed the appropriate one. Indeed, through restoring the complete 2D background spectrum specinholucy enables us to check the accuracy of the restoration at each pixel of the input 2D frame, or simply in the spatial (cross-dispersion) direction by comparing the collapsed spatial profiles of the input and restored 2D spectra. This is illustrated using real data in Fig. 12.

\section{The spatial resolution kernel}

The choice of the width of the resolution kernel for the background resides with the user. It is implemented as a Gaussian of user-chosen sigma $\sigma_{\text {kernel }}$ in the specinholucy code. It is difficult to give a general guide to its choice. Obviously it should be wider than the equivalent maximum Gaussian sigma of the SSF $\sigma_{\mathrm{SSF} \text { max }}$ in the cross-dispersion direction. The sigma of the kernel has to be tuned and is strongly dependent on the nature of the extended source and more specifically on the extended background component in the vicinity of the point source. Comparison of the restored background with the input spectrum will quickly show if narrow features in the extended background, above the noise, have failed to be modelled through choice of too wide a kernel width (see Fig. 5, left column). If the kernel width is too narrow then the point source will be partially modelled as a peak in the background channel and the fluxes in our restored point source spectrum will be underestimated (Fig. 5, right column). The optimal width for the spatial resolution kernel corresponds to a compromise between the lower spatial frequency of the extended background crossdispersion profile and the higher spatial frequency of the point source cross-dispersion profile. A decent first guess would be 


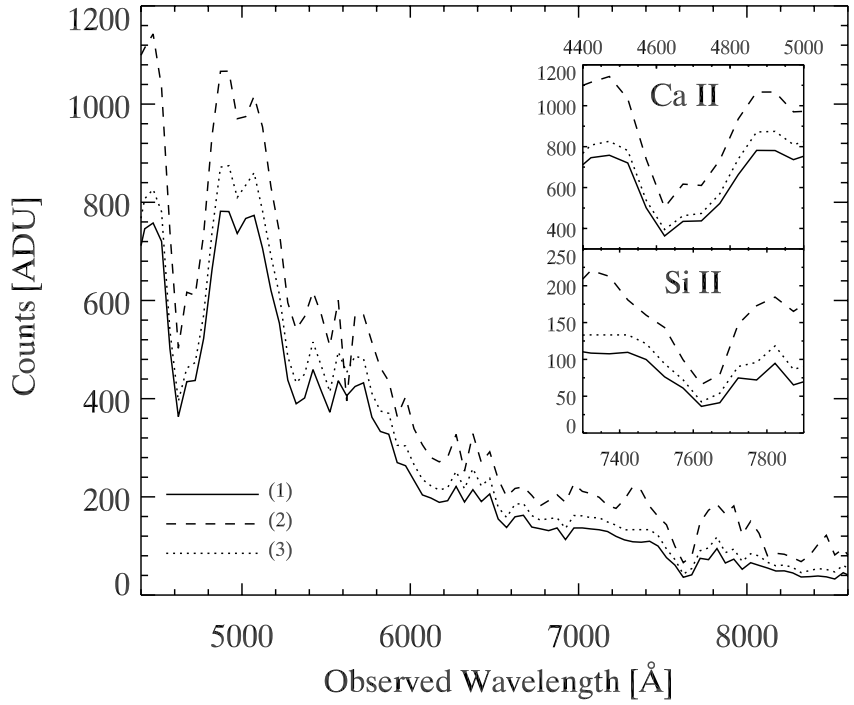

Fig. 4. Impact of the SSF on the restored point source spectrum of SN 2002go. Refer to Fig. 3 for the meaning of the line annotations (1), (2) and (3), noting that (1) corresponds to the polynomial fit to the SN 2002go profile. The two inlays zoom in on the blueshifted $\mathrm{Ca}$ II H\&K and Si II features, both prominent in SN Ia spectra, and highlight the impact of non-optimal spectral extractions on empirical correlations involving line strengths (see text).

$\sigma_{\text {kernel }} \approx 2-3 \overline{\sigma_{\text {SSF}}}$. In Fig. 5 (middle column) we have used $\sigma_{\text {kernel }} \approx 2.3 \overline{\sigma_{\mathrm{SSF}}}>\sigma_{\mathrm{SSF}, \max }$. From Fig. 5 we further note that, if one can clearly see the impact of a wide kernel on the spatial residuals $\delta_{\mathrm{gal}+\mathrm{SN}}$, discriminating between the narrow and optimal kernel widths - respectively 1.5 and 3.8 - is not obvious a priori. The optimal kernel width in Fig. 5 was chosen such as to maximise the counts in the point source channel, or $\delta_{\text {gal }}$, whilst keeping the spatial residuals $\delta_{\text {gal+SN }}$ below the statistical noise limit of the input 2D frame.

One important test is to ensure we are not capable of restoring a PSF-like spectrum from a pure extended source. This is particularly relevant to high- $z$ SN Ia spectroscopy since in many cases one is only able to extract a spectrum of the supernova host galaxy whereas one is convinced of having obtained a combined spectrum including the SN. Then the currently widespread technique of subtracting a galaxy template from this "combined" (and noisy) spectrum can sometimes reveal SN-like features where no supernova is present! This is illustrated later on in Sect. 7.3 where we compare the specinholucy output with that of a statistical algorithm $(\mathcal{S N}$-fit) which decomposes a 1D spectrum into its $\mathrm{SN}$ and galaxy components using a galaxy spectral template (see also Fig. 14).

Here we have used the uncontaminated galaxy spectrum of the host of SN 2002bo (see Sect. 6), obtained by constructing a mirror image of the portion of the galaxy profile devoid of supernova signal. We try to extract a point source component from this pure galaxy spectrum at different locations of the galaxy trace, including the centroid, using for this purpose three different SSFs of widths corresponding to the mean and extrema of the seeing values measured by the DIMM station for this observation. We then compare the restored point source flux with the statistical noise limit of the input 2D spectrum at

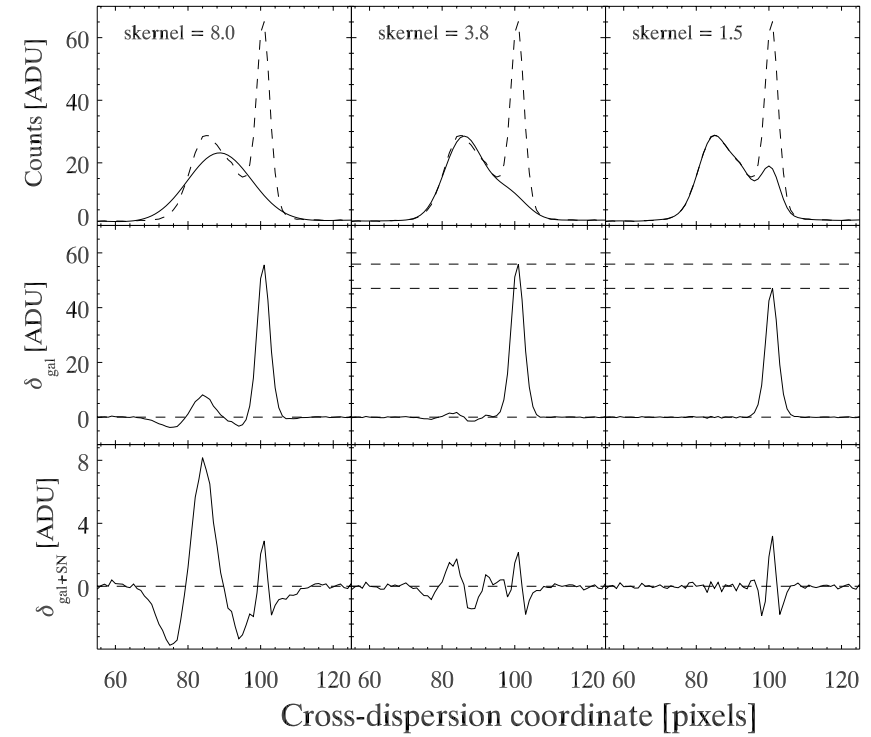

Fig. 5. Impact of the width of the spatial resolution kernel $\sigma_{\text {kernel }}$ on the background restoration. Top panel: input average (dashed line) and restored background (solid line) cross-dispersion profiles. Middle panel: residuals of the previous plot, revealing the point source channel (i.e. the supernova itself). Lower panel: spatial residuals of the input and restored 2D frames. Negative (positive) values in the residual plots mean we are over (under)-restoring the flux at that location.

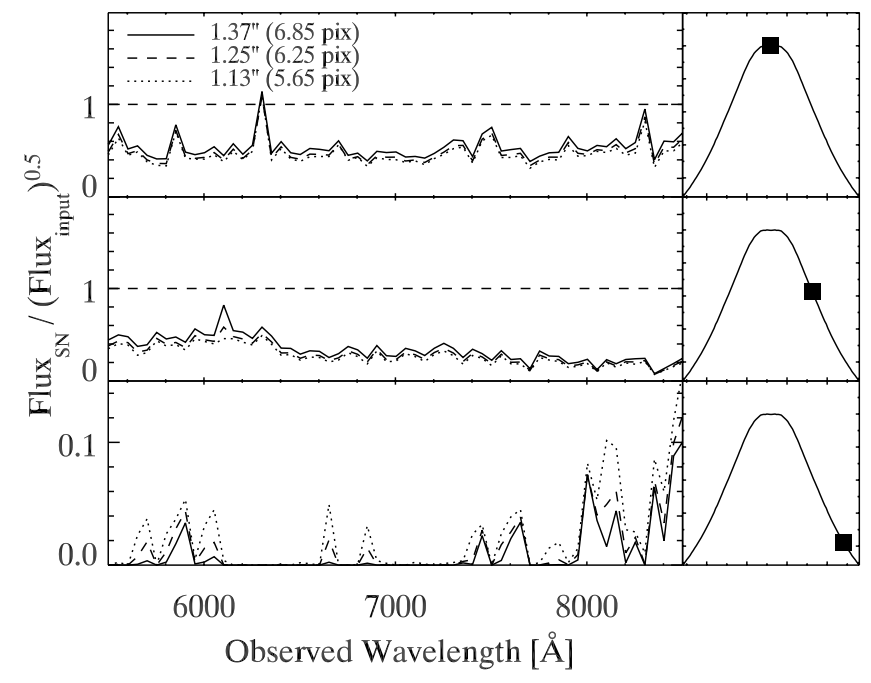

Fig. 6. Restored point source spectra in units of the statistical noise of the input pure galaxy spectrum. The different plots correspond to different positions of the point source to extract, marked by a filled square in the galaxy spatial profiles shown in the right column. The different lines correspond to different seeing conditions. The horizontal dashed line marks the limit below which no point source was detected in the background. Note the change of scale in the lower plot, where no flux at all is restored in many bandpasses. The peak above the dashed line in the uppermost plot is due to bad cosmic ray removal.

the position of the point source. Should this ratio fall below one this means that no point source was detected. We see from Fig. 6 that in all cases the restored spectrum is inconsistent with that of a PSF-like object over the whole spectral range. 
Table 2. Distances and flux scaling.

\begin{tabular}{llll}
\hline \hline Galaxy & $d$ (Mpc) & Reference & Factor $^{a}$ \\
\hline NGC 4526 & 13 & Drenkhahn \& Richtler (1999) & $1.5 \times 10^{-5}$ \\
NGC 6181 & 34 & Sil'chenko et al. (1997) & $1.1 \times 10^{-4}$ \\
\hline
\end{tabular}

${ }^{a}$ Factor used to scale down the flux, computed as $\left(d / d_{\mathrm{L}, z=0.5}\right)^{2} \times(1+z)^{-1}$.

\section{Testing the algorithm on simulated data}

In this section we present tests of the algorithm on simulated data, namely 2D spectra of a Type Ia supernova (SN Ia) at $z=0.5$ embedded in a late-type galaxy. We have chosen to reproduce a situation where a one hour-long exposure is taken at the ESO Very Large Telescope VLT-UT1 (8.2 m) using the FOcal Reducer Spectrograph (FORS1) in Long-Slit Spectroscopy (LSS) mode. The grism corresponds to $300 \mathrm{~V}$, and the slit width is $1^{\prime \prime}$. The reason behind chosing these specific settings is that they correspond to those used for the real data on which the algorithm will be tested further in Sect. 6. In what follows we assume a $\left(\Omega_{M}, \Omega_{\Lambda}, h\right)=(0.3,0.7,0.72)$ cosmology (where $h=\frac{H_{0}}{100 \mathrm{~km} \mathrm{~s}^{-1} \mathrm{Mpc}^{-1}}$ is the dimensionless Hubble constant), and so the luminosity distance corresponding to $z=0.5$ is $d_{\mathrm{L}, z=0.5} \sim 2.72 \mathrm{Gpc}$. The factors used to scale down the fluxes as the SN and galaxy are artificially redshifted to $z=0.5$ are listed in Table 2 . The additional factor of $(1+z)$ comes from the fact that we are integrating the flux over a finite bandpass, where the wavelength axis has been diluted by that same factor.

\subsection{Simulated data}

The simulated 2D spectra are a combination of a 2D supernova spectrum and a 2D background spectrum, itself consisting of a galaxy and a sky spectrum. We vary the phase of the SN, i.e. the brightness and the input spectrum, and its position within the galaxy from 0.75 to 1.75 , in units of $F W H M$ of the galaxy trace. Random poisson noise is added to the image using the gain and read noise characteristics of FORS1 so as to degrade the overall $\mathrm{S} / \mathrm{N}$ of the image and thereby reproduce plausible observing conditions.

\subsubsection{Supernova spectrum}

The 2D supernova spectra are synthesized using the 1D, fluxcalibrated spectra of the Type Ia supernova SN 1994D in NGC $4526\left(c z=448 \mathrm{~km} \mathrm{~s}^{-1}\right)$ as obtained from the SUSPECT database $^{3}$. Where spectral coverage at a given phase was lacking (i.e. at $-6 \mathrm{~d},+0 \mathrm{~d},+6 \mathrm{~d},+8 \mathrm{~d}$ and $+14 \mathrm{~d}$ ), approximate synthetic spectra were obtained from the two observed spectra closest in phase using the UBVRI photometry published in Patat et al. (1996). The same technique was applied to observed spectra whose wavelength range did not extend below $3650 \AA$ in the blue (at $-8 \mathrm{~d}$ and $+10 \mathrm{~d}$ ), so as to make sure to cover the Ca II H\&K (3934 ̊, $3968 \AA$ ) absorption trough blueshifted to $\sim 3750 \AA$, prominent in SNe Ia optical spectra (see Filippenko 1997 for a review). Each spectrum is then

\footnotetext{
${ }^{3}$ http://bruford.nhn.ou.edu/ suspect/
}

Table 3. SN 1994D data used in the simulation.

\begin{tabular}{lcccc}
\hline \hline Phase $^{a}$ & Date $^{b}$ & $m_{B}{ }^{c}$ & $m_{R, z=0.5}{ }^{d}$ & counts $^{e}$ \\
\hline-10 & $11 / 03 / 94$ & 13.26 & 24.86 & 13.5 \\
-8 & $13 / 03 / 94$ & 12.67 & 24.27 & 22.7 \\
-6 & $\ldots$ & 12.26 & 23.86 & 30.7 \\
-4 & $17 / 03 / 94$ & 11.99 & 23.59 & 37.4 \\
-2 & $19 / 03 / 94$ & 11.86 & 23.46 & 39.5 \\
+0 & $\ldots$ & $11.84^{\dagger}$ & 23.44 & 51.6 \\
+2 & $23 / 03 / 94$ & 11.89 & 23.49 & 48.4 \\
+4 & $25 / 03 / 94$ & 11.99 & 23.59 & 34.8 \\
+6 & $\ldots$ & 12.13 & 23.73 & 31.3 \\
+8 & $\ldots$ & 12.33 & 23.93 & 27.9 \\
+10 & $31 / 03 / 94$ & $12.55^{\dagger}$ & 24.15 & 21.5 \\
+12 & $02 / 04 / 94$ & 12.80 & 24.40 & 15.0 \\
+14 & $\ldots$ & 13.10 & 24.70 & 12.7 \\
\hline
\end{tabular}

${ }^{a} \mathrm{SN}$ phase in rest-frame days from $B$-band maximum.

${ }^{b}$ UT date of spectroscopic observation. Absence of date means the spectrum is synthetic.

${ }^{c} B$-band magnitude taken from Table 1 in Patat et al. (1996). Data marked with ${ }^{\dagger}$ results from a polynomial fit to the $B$-band light curve. ${ }^{d}$ The $R$-band at $z=0.5$ is close to rest-frame $B$-band, and so $m_{R}(z=0.5) \approx m_{B}-2.5 \log \left(\frac{d_{L, S N}}{d_{L, g a l}}\right)^{2} \approx m_{B}+11.60$.

${ }^{e}$ Mean total counts in the whole SN profile in ADUs per pixel along the dispersion axis.

redshifted to $z=0.5$ and rebinned to the pixel scale of FORS1 equipped with a $300 \mathrm{~V}$ grism and a $1^{\prime \prime}$ slit $\left(\sim 2.66 \AA \mathrm{pix}^{-1}\right)$. The flux (in units of erg s $\mathrm{s}^{-1} \mathrm{~cm}^{-2} \AA^{-1}$ ) is scaled down to take into account the change in luminosity distance as the source is artificially placed at $z=0.5$ - assuming SN 1994D to be at the same distance as its host galaxy - and is converted to counts (ADU) using a FORS1 sensitivity function. At this stage we have a 1D supernova spectrum in ADUs as a function of wavelength, corresponding to a one-hour integration on FORS1. Finally, a 2D supernova spectrum is synthesized by replicating this $1 \mathrm{D}$ spectrum along the lines of a 2D image array (i.e. along the dispersion axis) and multiplying each image column by a $1 \mathrm{D}$ Gaussian profile normalised to unity of FWHM corresponding to $1^{\prime \prime}$ seeing. Note that the flux vector is normalised to an airmass of 1, and no scaling was applied to account for observations (usually) made at higher airmasses.

The supernova data used in this simulation are summarised in Table 3 . The quoted $R$-band magnitudes are only approximate and are affected by systematic effects because of the lack of any $k$-correction. For comparison the mean number of counts (in ADUs) per pixel is 105.7 and 19.8 for galaxy and sky (including sky lines), respectively.

\subsubsection{Background spectrum}

For the galaxy spectrum we choose to artificially place SN 1994D in NGC 6181, an SC galaxy part of Kennicutt's spectrophotometric atlas of galaxies (Kennicutt 1992). The reason behind this is the prominent [O II] emission at $3727 \AA$ present in its spectrum (see Fig. 7), which could then serve as an diagnostic tool for probing the efficiency of our algorithm in restoring a clean point source spectrum. If successful, the 


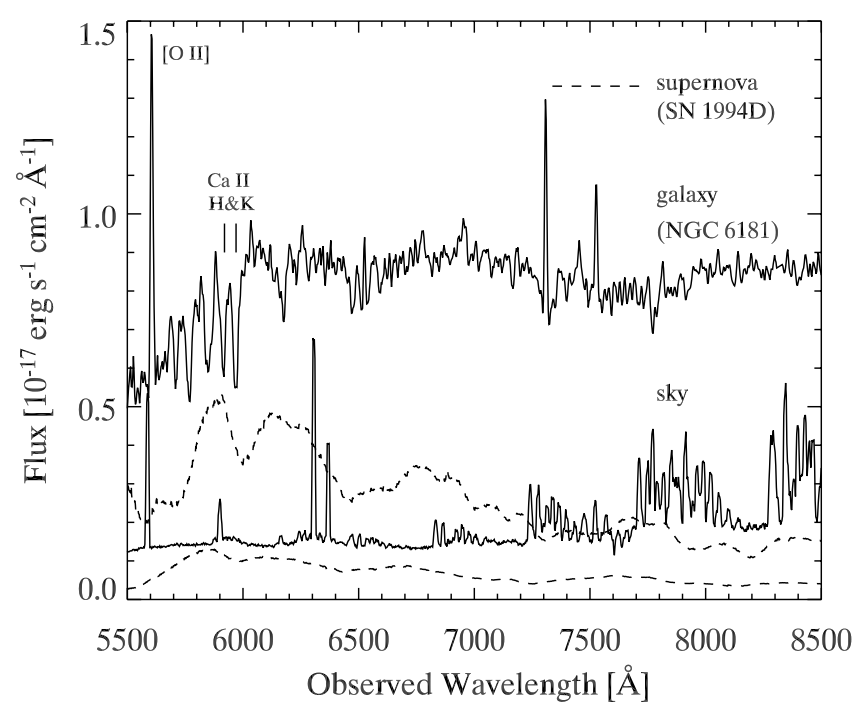

Fig. 7. Absolute flux-calibrated galaxy and sky spectra used in the simulation. The spectra are shown in observed wavelength at $z=0.5$, and without shot noise for sake of clarity. The prominent [O II] emission and the Ca II H\&K absorption in NGC 6181 are labelled accordingly. Overplotted (dashed lines) are the spectra of SN 1994D at maximum intensity (top) and ten days before maximum (bottom).

restored 1D spectrum of the point source should be devoid of [O II] flux residuals.

The spectrum of NGC 6181 was redshifted to $z=0.5$ and rebinned to the pixel scale of FORS1 using the same procedure as for the supernova spectrum. The flux was scaled down to take into account the change in luminosity distance, and converted to counts (again in ADUs) using the same sensitivity function as for the supernova. The resulting galaxy has a magnitude $m_{\text {gal }} \sim 21.5$ in a redshifted $B$-band filter (very roughly corresponding to the $R$-band at $z=0.5$ ) i.e. more than an order of magnitude brighter than when the supernova is at its faintest (see Table 3).

Next, a galaxy spatial luminosity profile was constructed based on a standard bulge+disk model. The bulge component is usually described analytically using the so-called $r^{1 / 4}$ law and the disk component follows an exponential decline with distance $r$ from the nucleus (de Vaucouleurs 1959; Freeman 1970). One can derive a global analytical form of the surface brightness profile $\Sigma_{\mathrm{S}}(r)$ of spiral galaxies, as a function of the effective radius $r_{\mathrm{e}}$ of the bulge only. In doing so we assume a bulge-to-total luminosity ratio $B / T=0.4$ (Ratnatunga et al. 1999) and a ratio between the effective radii of the bulge and disk components $r_{\mathrm{b}, \mathrm{e}} / r_{\mathrm{d}, \mathrm{e}}=0.5$ (Kent 1985; Ratnatunga et al. 1999):

$$
\begin{aligned}
\Sigma_{\mathrm{S}}(r)= & \Sigma_{\mathrm{b}}(r)+\Sigma_{\mathrm{d}}(r) \\
= & 0.76931 \Sigma_{\mathrm{S}, \mathrm{e}} \exp \left(-7.6692\left[\left(\frac{1.6617 r}{r_{\mathrm{e}}}\right)^{1 / 4}-1\right]\right) \\
& +2.9343 \Sigma_{\mathrm{S}, \mathrm{e}} \exp \left(-\frac{1.3945 r}{r_{\mathrm{e}}}\right),
\end{aligned}
$$

where $\Sigma_{\mathrm{b}}(r)$ and $\Sigma_{\mathrm{d}}(r)$ are the surface brightness profiles of the bulge and disk components, respectively, and $\Sigma_{\mathrm{S}, \mathrm{e}}(r)$ is the surface brightness at the effective radius $r_{\mathrm{e}}$.

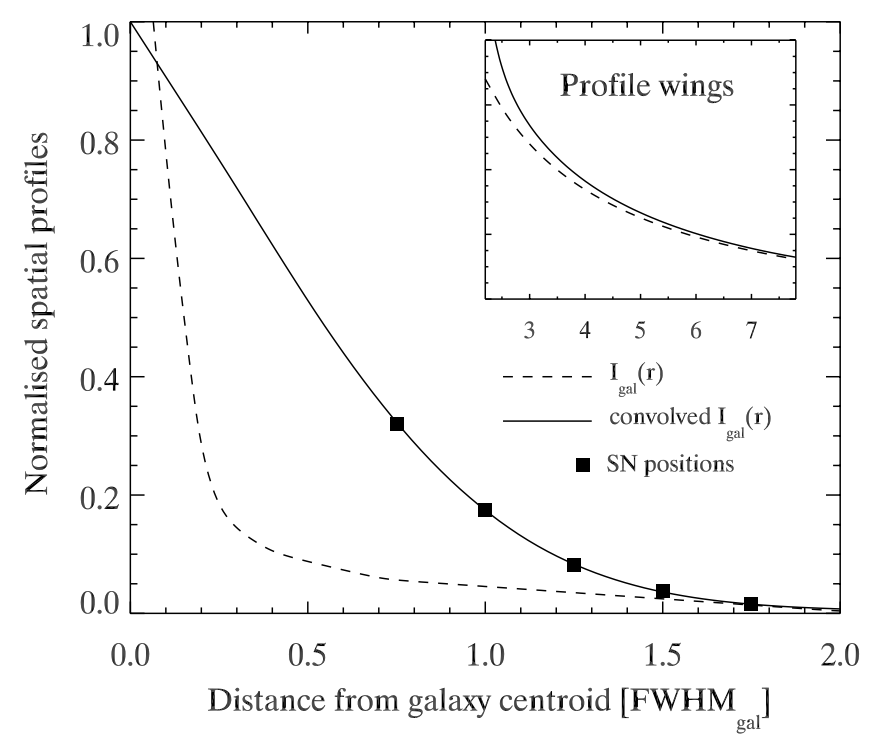

Fig. 8. Smoothed galaxy spatial profile used in the simulation, resulting from the convolution of a standard spiral galaxy luminosity profile and a $1^{\prime \prime}$ seeing Gaussian kernel. The abscissa is in units of FWHM of the convolved galaxy profile ( $\cong 3.06$ pix). The effective radius $r_{\mathrm{e}}$ in these units is at $\sim 7.36$. Filled squares indicate the position of the SN used in this simulation, namely $0.75,1.00,1.25,1.50$ and 1.75 .

The angular size of NGC 6181 is $2.5^{\prime} \times 1.1^{\prime}$ (NASA/IPAC Extragalactic Database ${ }^{4}$ ), which corresponds to $4.5^{\prime \prime} \times 2.2^{\prime \prime}$ at $z=0.5$, and in turn to $22.5 \times 11$ pixels on a FORS1 image. Assuming the slit was placed along the major axis of NGC 6181 the effective radius of the disk $r_{\mathrm{e}}$ is 22.5 pixels. The derived luminosity profile is then normalised to unity and is multiplied into each column of the 2D galaxy image array. Each column is further convolved with a Gaussian profile of FWHM corresponding to $1^{\prime \prime}$ seeing to reproduce similar observing conditions as for the supernova. The resulting spatial profile $I_{\text {gal }}(r)$ is shown in Fig. 8. Notice how at these redshifts the core spatial profile of galaxies is entirely seeing-dominated, and one must rely on the broad extended wings of the profile to differentiate between a galaxy and a point source.

The sky 2D spectrum (the second background component for ground-based observations) is generated by uniformly replicating an observed 1D sky spectrum with the required settings (300V grism, 1" slit) along lines of an image array, and scaled to the required exposure time ( $3600 \mathrm{~s}$ in our case). It is thus by construction perfectly flat and monochromatic in the spatial direction. We do not have to worry about spatial distortions of sky lines, and sky background removal in the input 2D spectrum is rendered trivial.

\subsection{Simulation steps}

We now move on to outlining the steps made in carrying out the simulation. Far from being redundant these are the same steps that one should in principle go through when applying this technique to real data. They include determining the position (and slope) of the supernova spectral trace, its FWHM as a

\footnotetext{
${ }^{4}$ http://nedwww.ipac. caltech.edu/
} 
function of wavelength, synthesizing a corresponding SSF and running the specinholucy code with the optimal settings. For the purpose of this simulation and for ease in computing statistics these steps have been made automatic, though we strongly advise to make them highly interactive when dealing with real data.

\subsection{1. $\mathrm{SN}$ trace position and slope}

One of the inputs to the specinholucy routine is a position table containing the $\left(x, y\right.$, slope $\left.\mathrm{pix}^{-1}\right)$ of the supernova spectral trace. Since in the case of high- $z$ SNe Ia we are limited in S/N we cannot simply give an approximate position and rely on cross-correlations with the SSF to determine a more accurate one. Here we must input the exact SN trace position to which the SSF profiles will be shifted. The results of our simulation (see Sect. 5.3) show the impact of positional accuracy on the point source restoration.

To some extent one should base the method used to determine the trace position on the characteristics of the instrument used for the observation. In the case of FORS1 we know from observations of standard stars that the position of a point source does not vary by more than one pixel across the whole CCD chip. Thus, a $\sim 1$ pix accuracy can almost routinely be achieved by summing up each of the spatial channels and finding the maximum intensity (other than the galaxy) in a given cross-dispersion region. Clearly this will be problematic for cases where the $\mathrm{SN}$ is close to the centroid of the galaxy trace or when its phase is far from maximum $(-10 \mathrm{~d}$ or $+14 \mathrm{~d})$. Alternatively one can try to fit the background around the SN trace and subtract this fit from the overall profile. The residuals can then be fit with a Gaussian profile and the trace position is taken to be the profile centre.

The result of this operation is a relation between $\mathrm{SN}$ trace position and dispersion coordinate $x$, which is fit linearly so as to determine the trace position and slope. We now have our input $\left(x, y\right.$, slope pix $\left.^{-1}\right)$ table necessary to run specinholucy. A plot of the error made in determining the trace position using this approach is shown in Fig. 9. The position is best determined when the signal-to-noise ratio is high, namely when the contrast is high between the $\mathrm{SN}$ and the underlying background, as expected.

\subsubsection{SN trace FWHM and SSF synthesis}

To construct a synthetic SSF we need to know the FWHM of the $\mathrm{SN}$ trace as a function of the dispersion coordinate. We proceed in a way analogous to determining the $\mathrm{SN}$ trace position, except we are now interested in the width of the Gaussian fit to the residual signal left over after sky and galaxy subtraction rather than the Gaussian centre. A similar relation between $F W H M_{\mathrm{SN}}$ and dispersion coordinate is established and a table of ( $F W H M_{\mathrm{SN}}$, wavelength) values is elaborated and serves as input for the SSF synthesis using specpsf. A plot of the error made in determining the trace $F W H M$ using this approach is shown in Fig. 10. Again, the FWHM is better determined for cases with a higher SN flux relative to the galaxy background.

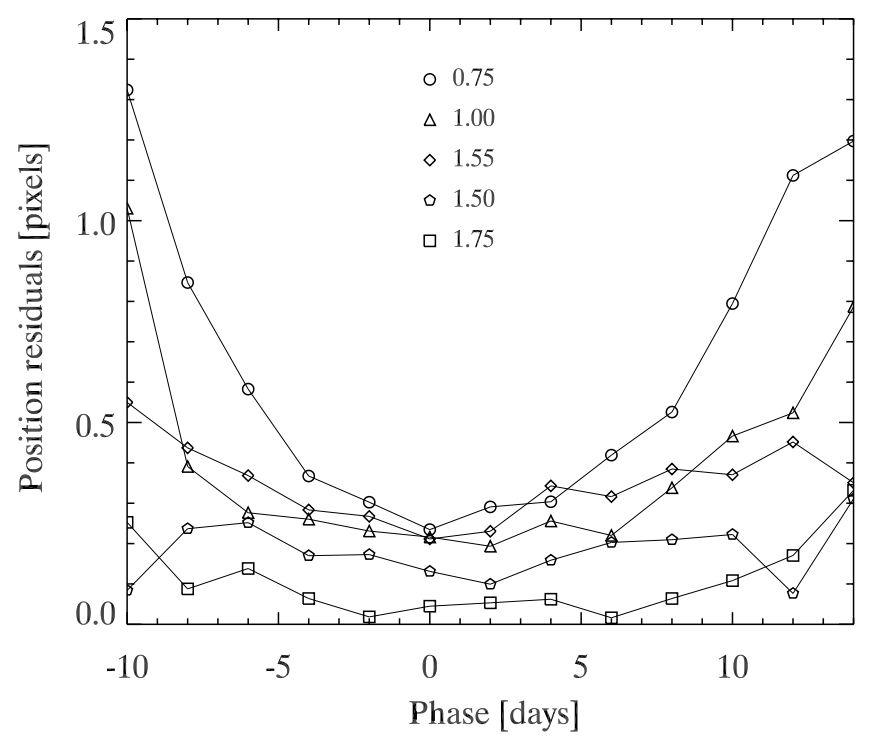

Fig. 9. SN trace position residuals as a function of the SN phase, for different locations of the SN with respect to the galaxy centroid. Each curve corresponds to a specific SN position, given in units of $F W H M_{\text {gal }}$.

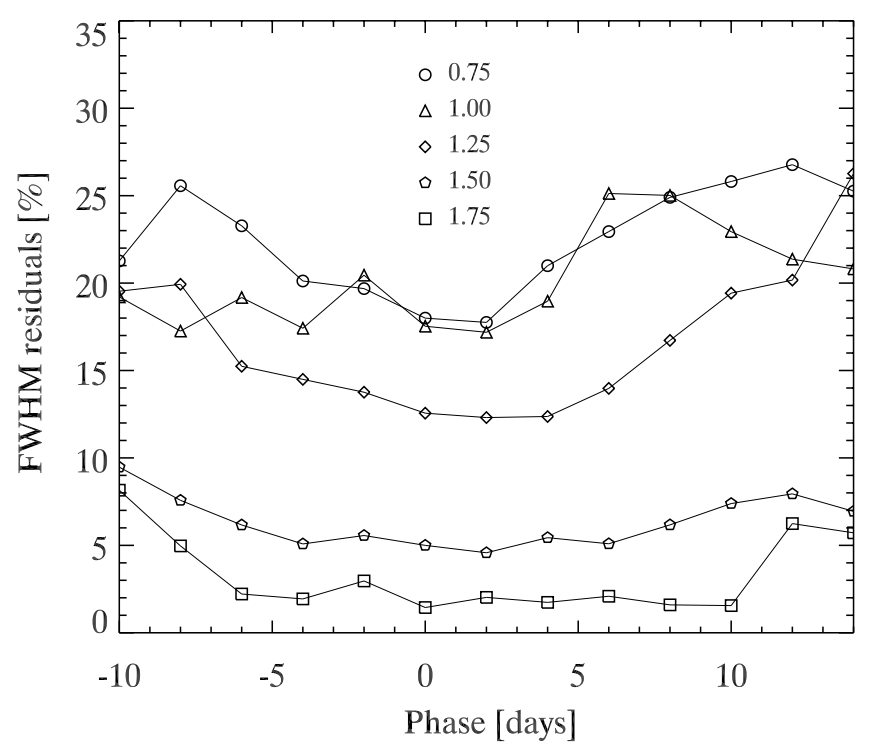

Fig. 10. SN trace $F W H M$ residuals as a function of the SN phase, for different locations of the $\mathrm{SN}$ with respect to the galaxy centroid. The curves and plotting symbols have the same meaning as in Fig. 9.

\subsubsection{Running specinholucy}

Once we have determined the position (and slope) of the SN trace and synthesized an SSF spectrum using its FWHMwavelength dependency, we can run specinholucy on the input sky-subtracted 2D spectrum. We refer the reader to Lucy \& Walsh (2003) and to the IRAF help pages for the specinholucy task for a more complete description of the input parameters. A centroid fitting is used to determine the SSF peak at each column, and no subsampling (in the spatial direction) is performed since the resulting PSF profile is not oversampled with respect to the input $2 \mathrm{D}$ spectrum. A cubic spline is used as the interpolation method to shift the SSF to the position of the point 
source - which is defined exactly in the input position table. Note that no input statistical image to determine the statistical error at each pixel is used in these simulations.

The only input parameter made to vary from one run to the other is the width $\sigma_{\text {kernel }}$ of the Gaussian used for the spatial resolution kernel. We see from Fig. 8 that the galaxy spatial profile is entirely seeing-dominated, meaning that the widths of the SN and galaxy traces are comparable at each wavelength. This in turn implies that the spatial frequency of the galaxy profile will vary hugely when varying the SN position from 0.75 to $1.75 F_{W H M_{\text {gal }}}$. To avoid confusion in the point/extended source discrimination we must use a narrow (wide) spatial resolution kernel when the $\mathrm{SN}$ is close to (far from) the galaxy centroid, where the spatial frequency is high (low).The input values for the smoothing kernel were chosen such as to minimise the mean flux residuals in restoring all phases of the SN spectrum at a given position. For all runs the convergence criteria for the SN and background spectra per $R-L$ iteration (see Sect. 2) were set to $0.1 \%$ and $1 \%$, respectively.

Two runs (A and B) were executed: in run A we use the $\mathrm{SN}$ position and $F W H M$ as determined using the method outlined in Sects. 5.2.1 and 5.2.2, whilst in run B we use the known values of the $\mathrm{SN}$ trace position and $F W H M$ to synthesize the SSF and restore the SN spectrum. This enables us to investigate the joint impact of the errors in the SN position and FWHM on the accuracy of the SN spectral restoration (see Sect. 5.3).

\subsubsection{Statistical calculation}

To evaluate the efficiency of the method we compare the (known) input SN spectrum with its restored version. More specifically we compute the ratio of the flux residuals with the statistical variation in the input $2 \mathrm{D}$ spectrum. Should this ratio fall below one this means that we are statistical noise-limited and cannot improve on the restoration.

In this simulation we compare the flux residuals both over the whole observed spectral range (5500 $⿱-8500 \AA)$ and around the region of host galaxy [O II] $3727 \AA$ emission. This region is simply defined in wavelength space (in $\AA$ ) as $3727(1+z) \pm F W H M_{[\mathrm{O} I \mathrm{I}]}(1+z)$, where $F W H M_{[\mathrm{O} \text { II }]}$ is the width of the [O II] line in the input non-redshifted spectrum of NGC 6181 and $z=0.5$ is the redshift of the simulation. For both cases the flux residual $\delta F$ is evaluated at each pixel according to:

$\delta F=\frac{\overline{\mid F_{\mathrm{SN}, \mathrm{i}}-F_{\mathrm{SN}, \mathrm{r}}} \mid}{\sqrt{F_{\mathrm{SN}, \mathrm{i}}+F_{\mathrm{gal}, \mathrm{i}}+F_{\mathrm{sky}, \mathrm{i}}}}$,

where $F_{\mathrm{SN}, \mathrm{i}}$ and $F_{\mathrm{SN}, \mathrm{r}}$ are the input and restored $\mathrm{SN}$ fluxes, respectively. $F_{\text {gal,i }}$ and $F_{\text {sky,i }}$ are the galaxy and sky fluxes at the location of the $\mathrm{SN}$, defined as an interval centred on the exact $\mathrm{SN}$ trace position and of width the $F W H M$ of the $\mathrm{SN}$ trace. It is important to compute the statistical noise as that in the total signal at the position of the supernova (i.e. including the underlying background) and not just in the SN signal itself. If $\delta F \leq 1$ one can in principle consider the $\mathrm{SN}$ restoration to have converged.

\subsection{Simulation results and discussion}

The simulation results are summarised in Fig. 11. Both runs A and B (see Sect. 5.2.3) are shown in order to evaluate the impact of the error made in the determination of the SN trace position and $F W H M$ on the restoration of the supernova spectrum.

These plots clearly show that the limiting factor of this twochannel restoration technique (as for any other spectral extraction method) is the contrast between the point source and the underlying background. The overall flux is systematically restored to the statistical noise limit when the supernova is at its brightest stages ( $-5 \mathrm{~d} \lesssim$ phase $\lesssim+5 \mathrm{~d}$ ), irrespective of its position with respect to the centroid of the galaxy trace. In this phase regime the error in the SN trace position and FWHM is also at its lowest (see Figs. 9 and 10) and has little impact on the quality of the restoration - as shown by the dashed lines in Fig. 11. This is not always the case for the [O II] flux residuals, where the added background noise due to the presence of this emission line affects the restoration (cf. at positions 0.75 and 1.00 , in units of $\left.F W H M_{\mathrm{gal}}\right)$.

One sees the dramatic impact of the error made in the $\mathrm{SN}$ trace position and $F W H M$ at low $\mathrm{S} / \mathrm{N}$, namely when the $\mathrm{SN}$ is outside the $-5 \mathrm{~d} \lesssim$ phase $\lesssim+5 \mathrm{~d}$ range and close to the centroid of the galaxy (at positions of $0.75,1.00$ and 1.25). For errors in the position $\gtrsim 0.3$ pix and $F W H M_{\mathrm{SN}} \gtrsim 15 \%$, the restoration fails to reach the statistical noise limit. These are the main sources of systematic error in the restoration method and great care should be taken when determining the SN trace position and FWHM when applying it to real data.

\section{Testing the algorithm on real data}

In this Sect. we apply this restoration technique to real supernova data. An outline of the steps involved (determination of the SN trace position and FWHM; SSF synthesis; running of specinholucy) has already been given in Sect. 5.2. We simply provide the input parameters that were used to run the code (see Table 4). All the data were collected by the authors via the ESO observing programme 170.A-0519 and are available through the ESO Science Archive Facility ${ }^{5}$. The observations were made with VLT+FORS1 in Long Slit Spectroscopy (LSS) mode with a $300 \mathrm{~V}$ grism and a $1^{\prime \prime}$ slit. The results of the twochannel restoration are shown in Figs. 12 and 13, whilst the individual objects are presented in Table 5 . On average $\sim 150$ iterations were needed to reach convergence for the restoration of the point source spectra, corresponding to $\sim 2 \mathrm{~min}$ on a $2.4 \mathrm{GHz}$ Pentium 4 processor.

There is one important distinction to be made in the calculation of the flux residuals here as opposed to that made for the simulation in Sect. 5.2.4. For observed cases we do not know how the total flux $F_{\text {tot }}$ separates into the individual components $F_{\mathrm{SN}}, F_{\text {gal }}$ and $F_{\text {sky }}$, and so our evaluation of the accuracy of the restoration is restricted to the calculation of the mean total flux residuals $\delta F_{\text {tot }}$ :

$\delta F_{\text {tot }}=\frac{\overline{\left|F_{\text {tot }, \mathrm{i}}-F_{\text {tot, } \mathrm{r}}\right|}}{\sqrt{F_{\text {tot }, \mathrm{i}}}}$.

5 http://archive.eso.org/ 

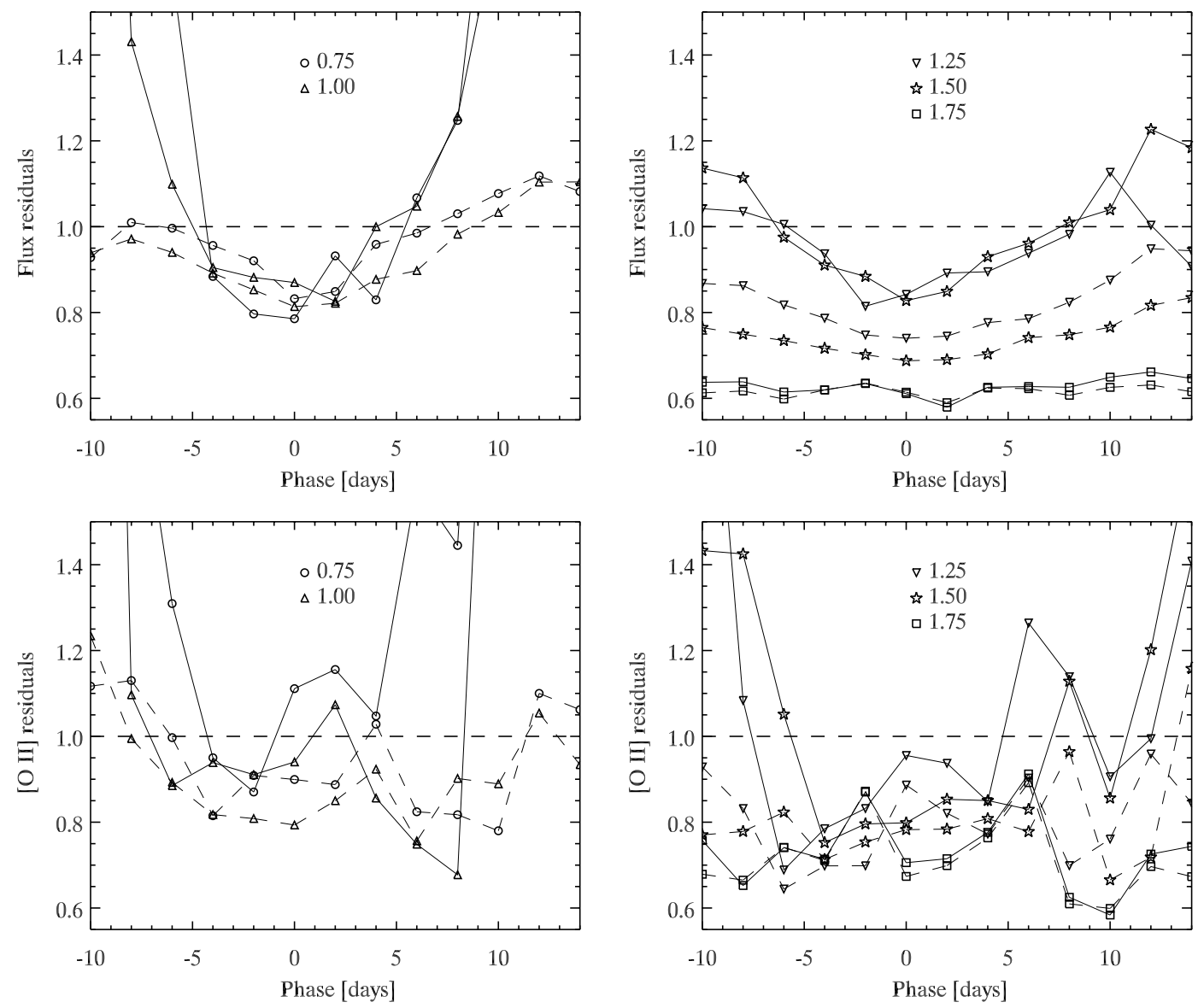

Fig. 11. Top panel: flux residuals $\delta F$ as a function of the SN phase for different positions of the SN with respect to the centroid of the galaxy trace, given in units of $F W H M_{\text {gal }} . \delta F \leq 1$ means the $\mathrm{SN}$ spectrum has been restored to the statistical noise limit. The solid and dashed lines correspond to runs A and B, respectively (see Sect. 5.2.3). Lower panel: [O II] flux residuals as a function of phase for different SN positions.

This is not as limiting as it may seem since we are again able to quantitatively evaluate our combination of $\sigma_{\text {kernel }}$ and $\sigma_{\mathrm{SSF}}$ through maximising the counts in the point source channel whilst ensuring that $\delta F_{\text {tot }}<1$ (cf. Sect. 4).

Figure 12 illustrates what has been said about an a posteriori check of the adequacy of both the SSF and the spatial resolution kernel. In all cases we not only fit the SN trace but also the underlying background such that the flux residuals $\delta F_{\text {tot }} \leq 1$. For the case of SN 2002 bo a secondary point source (indicated by an arrow) in its vicinity had to be included in the input position table - and hence allocated to the point source channel - for the restoration to succeed.

Figure 13 on the other hand shows the flux residuals in the dispersion direction, and one can immediately appreciate the successful allocation of (extended) atmospheric absorption features and sky emission lines to the background channel. Moreover one can immediately pick out the spectral regions affected by systematic errors in the restoration $\left(\delta F_{\text {tot }}>1\right)$ and only use those where $\delta F_{\text {tot }} \leq 1$ for analysis. The (spectral) residuals in this case were evaluated in the dispersion direction by evaluating the spatial residuals in a region centred on the SN position and of extent $3 \sigma_{\mathrm{SSF}}$. This ensures that we are only including the point source and its immediately underlying background.
Table 4. Specinholucy parameters.

\begin{tabular}{lcccll}
\hline \hline $\mathrm{SN}^{a}$ & $\sigma_{\text {kernel }}$ & \multicolumn{2}{c}{$F W H M$} & $\mathrm{SN}+\mathrm{bg}^{c}$ & $\mathrm{SN} / \mathrm{bg}^{d}$ \\
& [pixels] & DIMM & SSF & & \\
\hline 2002bo_1 & 4.0 & 6.25 & 6.93 & 63.6 & 4.8 \\
2002bo_2 & 4.0 & 7.95 & 6.57 & 62.5 & 4.6 \\
2002gr & 2.2 & 5.85 & 3.46 & 9.0 & 1.4 \\
2002go & 3.5 & 5.75 & 3.97 & 5.2 & 2.1 \\
\hline
\end{tabular}

${ }^{a}$ IAU designation. Note that two spectra were taken of SN 2002 bo. ${ }^{b}$ Mean FWHM in pixels of the Gaussian seeing corresponding to the DIMM station measurement or to the SSF used to extract the spectrum.

${ }^{c}$ Mean number of counts (ADU) in the dispersion direction of the input 2D spectrum at the location of the SN.

${ }^{d}$ Ratio of the integrated SN flux and that of the underlying background. One can calculate the mean signal-to-noise per pixel using SNR pix $^{-1}=\mathrm{SN} / \mathrm{bg}+1$.

\section{Comparison with other methods}

We choose to compare our method with alternative techniques, namely a standard extraction using IRAF, an iterative Gaussian extractor and a statistical decomposition of a 1D flux-calibrated spectrum into SN and galaxy contributions. The reason for including the latter (1D) approach is to highlight the need for the 


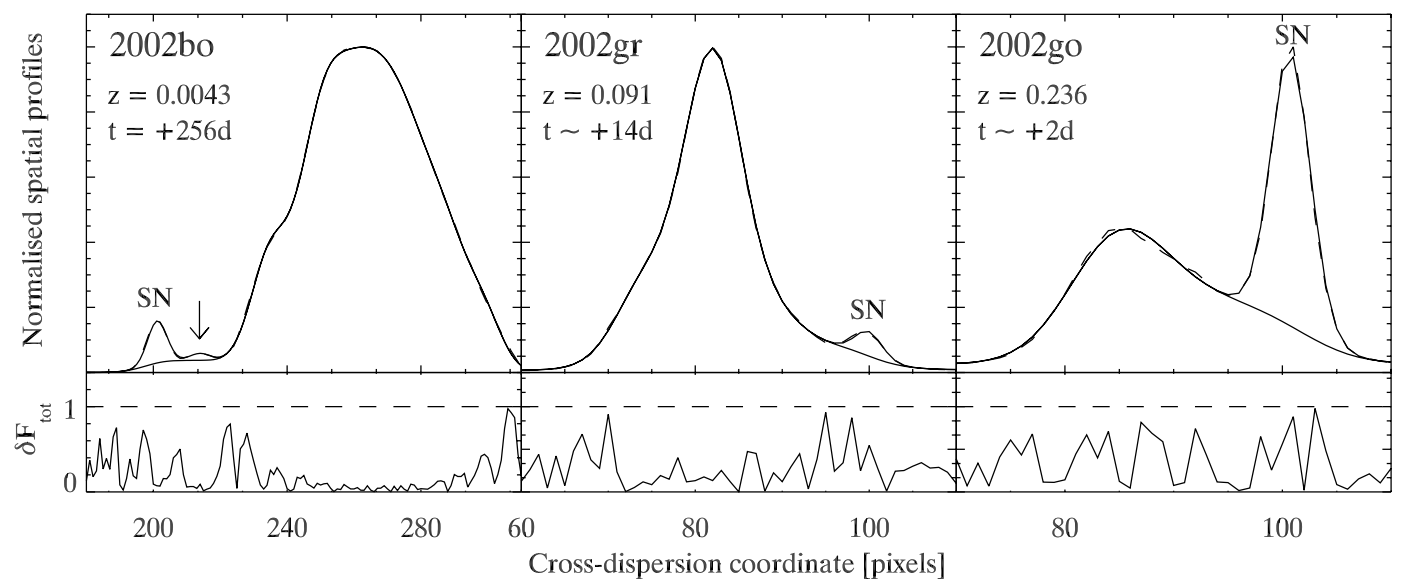

Fig. 12. Top panel: normalised average spatial profiles of the input (dashed line) and restored (solid line) 2D background spectra. Two runs of specinholucy were executed, one including the point source in the restored 2D background spectrum and the other excluding it, so as to appreciate how well the background underneath the SN was fit. Lower panel: wavelength-averaged spatial residuals in units of the statistical noise of the input $2 \mathrm{D}$ spectrum. For all cases we have $\delta F_{\text {tot }} \leq 1$, meaning the combined $\mathrm{SN}+$ background spatial profile is restored to the statistical noise limit over the whole spatial range.

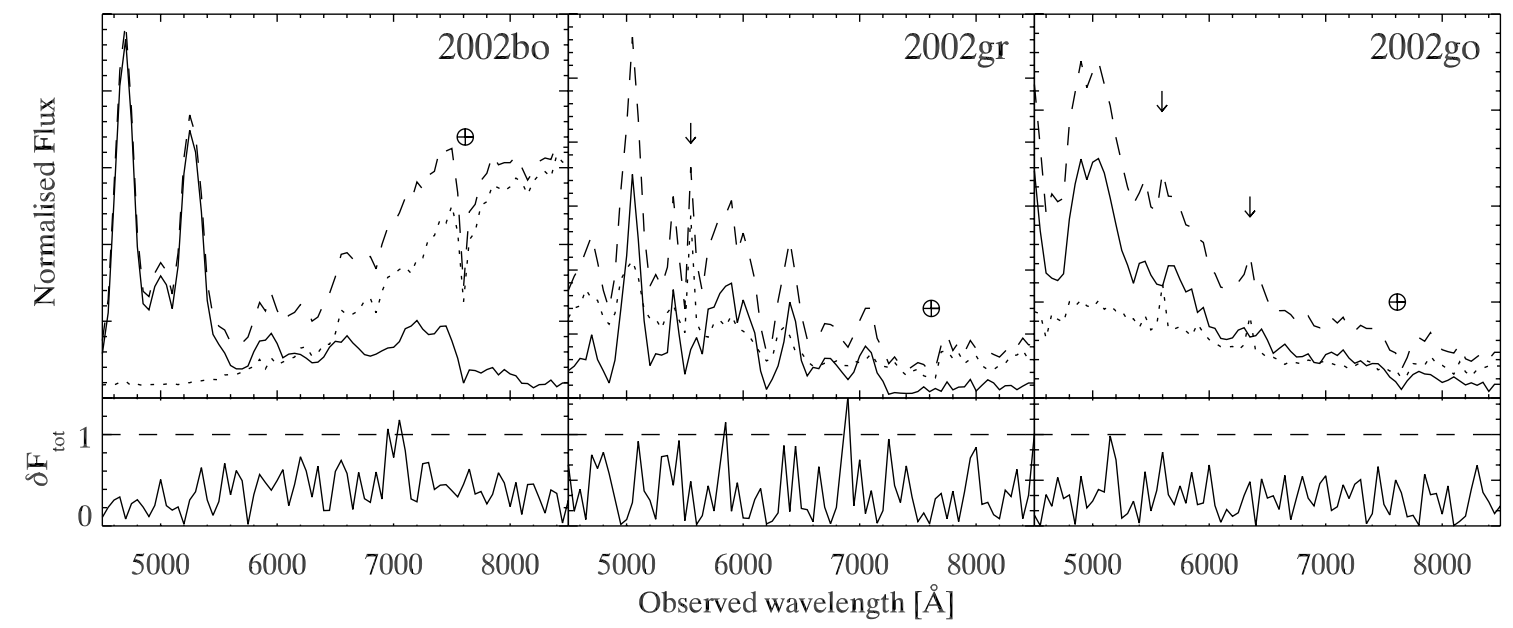

Fig. 13. Top panel: normalised restored point source spectra (solid line) and underlying background, both including (dashed line) and excluding (dotted line) the point source. The spectra have been normalised to the integral of the underlying background flux. The symbol $\oplus$ denotes the atmospheric $A$-band ( 7600-7630 ̊), whilst arrows indicate strong sky emission lines. Lower panel: spectral residuals in units of the statistical noise of the input $2 \mathrm{D}$ spectrum.

Table 5. Supernova observation summary.

\begin{tabular}{lllll}
\hline \hline SN $^{a}$ & Archive id. & UT date & $z^{c}$ & Phase $^{d}$ \\
\hline 2002bo & 2002bo & 06 Dec. 2002 & 0.0042 & +258 \\
2002gr & sloan9 & 11 Oct. 2002 & 0.091 & $+14^{\dagger}$ \\
2002go & sloan5 & 11 Oct. 2002 & 0.236 & $+2^{\dagger}$ \\
\hline
\end{tabular}

${ }^{a}$ IAU designation.

${ }^{b}$ Target name for search in the ESO archive.

${ }^{c}$ Redshift determined from nebular lines in the host galaxy.

${ }^{d} \mathrm{SN}$ phase in rest-frame days from $B$-band maximum. Phases marked with ${ }^{\dagger}$ have been determined from cross-correlations with local SN Ia templates using the SuperNova IDentification (SNID) code (Tonry et al. 2005).

SN-galaxy separation to occur at the earliest stages of the reduction process, and not as part of a post-processing chain of data analysis. The results are summarised in Fig. 14. Note that algorithms similar to ours do exist (though unlike ours they are not publicly available), and should these be of interest to the reader we refer him/her to Lucy \& Walsh (2003), Sect. 6.

\subsection{Standard extraction in IRAF}

For most purposes IRAF and similar reduction software are sufficient for extracting a high $\mathrm{S} / \mathrm{N}$ supernova spectral trace. The extraction can be further optimised by e.g. tracing the point source signal along the dispersion direction or varianceweighting the output flux. However, the hold on systematic errors is null and one tends to evaluate the resulting spectrum on purely qualitative grounds. In the case of high- $z$ SN Ia spectroscopy the extraction of the SN spectrum is often considered successful when the cross-correlation with a local SN Ia template spectrum is maximal. This clearly bypasses the specificity of the high- $z$ spectrum and affects the search for systematic 


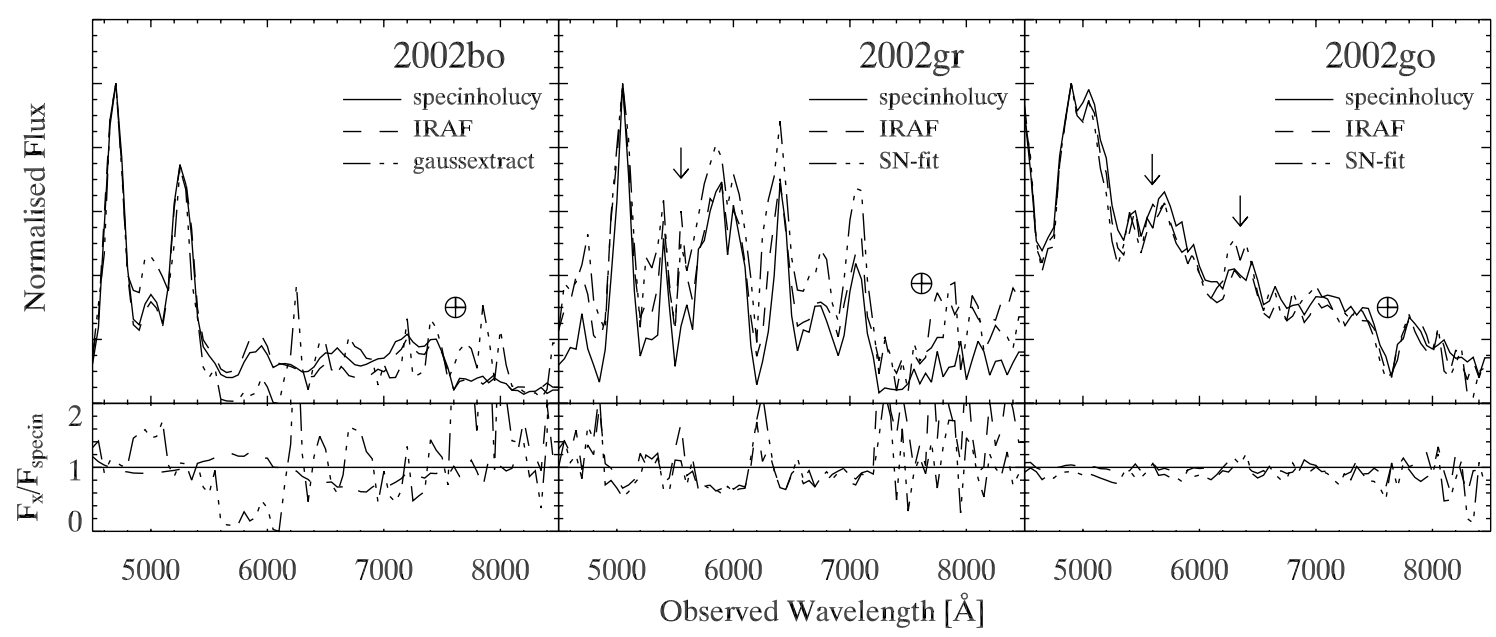

Fig. 14. Top panel: restored supernova spectra using specinholucy (solid line) and other methods presented in Sect. 7. The spectra are normalised to the maximum flux value of the specinholucy output. Due to the specificities of $\mathcal{S N}$-fit - namely the restriction of the local SN template spectra to epochs $-20 \mathrm{~d}<t<+20 \mathrm{~d}$, we restrict its application to SNe 2002gr and 2002go (we plot the solution corresponding to the smallest $\chi^{2}-$ see text.), for which we have not plotted the gaussextract output for the sake of clarity. The symbol $\oplus$ and arrows have the same meaning as in Fig. 13. Lower panel: ratios of the various spectra to the specinholucy output. We have deliberately restricted the $y$-axis range to [0,2] to be able to visualise small $\sim 20 \%$ differences in the SN flux.

spectral differences between high-z SN Ia spectra and their local counterparts.

Of equal concern is the arbitrary definition of the SN "background" in IRAF. One specifies a set of spatial coordinates in the vicinity of the SN trace to be fit by some user-defined polynomial ... and essentially hopes for the best! In particular one has to find a compromise between the varying width of the SN cross-dispersion profile (i.e. its $F W H M$-wavelength relation) and the varying structure of the underlying background. Cases such as that encountered for SN 2002bo - namely the contamination of the close $\mathrm{SN}$ background by a secondary point source - are extremely delicate to handle in a coherent way with standard spectral reduction packages and lead in this case to $\gtrsim 40 \%$ errors in the SN flux. Other errors in the extraction using IRAF are due to the varying spectrum of the underlying background, to strong sky emission lines and to the overall increase in sky noise at long wavelengths. The extracted spectrum of SN 2002go for instance is clearly affected by sky residuals, and more specifically the Si II feature (6355 $⿱$ ) blueshifted to $\sim 6150 \AA$ and characteristic of Type Ia supernovae lies in the same region as the atmospheric $A$-band at $z=0.236$, and is clearly contaminated by it. Any measure of line strengths and ratios in this region will thus also be affected, leading to significant errors in empirical correlations based on such measurements, e.g. the "Nugent relation" (see Nugent et al. 1995; and Sect. 3.3 in this paper). In cases where the signal-to-noise ratio is low (cf. SN 2002gr) there is obvious contamination from the underlying background in the SN spectrum, leading to significant errors; in fact, in many bandpasses no SN flux is output at all, where specinholucy is able to restore the $\mathrm{SN}$ contribution over the whole wavelength range.

\subsection{Gaussextract - an iterative Gaussian extractor}

In applying specinholucy to the restoration of supernova spectra we need to degrade the resolution of the background channel to discriminate between the $\mathrm{SN}$ and its underlying background. To illustrate the advantages of using a twochannel restoration we have written a software (gaussextract) that iteratively extracts the point source spectrum using the same synthetic SSF as for the specinholucy run. As in specinholucy, each dispersion channel is treated independently and the process is iterated till some minimal fractional change in the restored SN flux is achieved. For the sake of comparison with specinholucy, gaussextract runs the same convergence criteria for the point source flux. The difference here is that the SN and underlying background are jointly extracted in the same channel, leading to significant contamination by sky lines and host galaxy in the output SN spectrum (cf. SN 2002bo in Fig.14).

\subsection{Statistical approach using $\mathcal{S N}$-fit}

$\mathcal{S N}$-fit is a software package developed by Grégory Sainton (see Sainton 2004) to rapidly identify the supernova type and redshift in the SuperNova Legacy Survey (SNLS). The goal is to quickly identify candidates from their spectrum, for which the SN phase and the fraction of host galaxy light can be estimated. The algorithm is based on a $\chi^{2}$ fitting of the observed (and reduced) 1D spectrum to known template spectra. More specifically the observed spectrum is compared with a model made of a fraction $\alpha$ of SN $(\mathcal{S})$ and a fraction $\beta$ of galaxy $(\mathcal{G})$. The following model spectra $\mathcal{M}$ are built :

$\mathcal{M}\left(\lambda_{\text {rest }}\right)(z, \alpha, \beta)=\alpha \mathcal{S}\left(\lambda_{\text {rest }}(1+z)\right)+ \begin{cases}\beta \mathcal{G}\left(\lambda_{\text {rest }}(1+z)\right) & \text { (a) } \\ \beta \mathcal{G}\left(\lambda_{\text {obs }}\right), & \text { (b) }\end{cases}$

where $\lambda_{\text {rest }}$ and $\lambda_{\text {obs }}$ are the rest-frame and observed wavelength, respectively; (a) corresponds to the case where a local galaxy template spectrum is redshifted to fit the observed spectrum, whereas (b) makes use of the observed SN host galaxy itself. The $\chi^{2}$ is made robust against aberrant points (remaining sky lines, mostly). The fit is done for every galaxy-supernova pair and the results are sorted in ascending $\chi^{2}$. One is therefore 
able to evaluate how significant the best result is with respect to other SN-galaxy combinations, and in turn how confident one should be about the output supernova type, redshift and epoch.

It should be noted that, contrary to the other methods presented in this section, $\mathcal{S N}$-fit is a post-processing tool and thus cannot be required to correct for systematic errors made in the reduction process (most likely using standard software packages such as IRAF or MIDAS ${ }^{6}$ ), such as improper removal of sky lines. These errors will propagate in the $\mathcal{S N}$-fit result, as seen in the output spectrum of SN 2002go where the Si II feature is also affected by the atmospheric $A$-band. SN $2002 \mathrm{gr}$ on the other hand illustrates the limits of using a $\chi^{2}$ minimisation technique to separate the SN from the underlying background in low signal-to-noise cases.

The advantages of this purely statistical approach is that no a priori assumption is made about the underlying background. The host galaxy contamination is removed using a galaxy template or better still the SN host galaxy itself. The determination of the supernova type and redshift is greatly improved upon and the enhanced accuracy of this approach is discussed in Sainton (2004). The disadvantage is that we are limited by the database of local $\mathrm{SNe}$, and any spectral peculiarities will not be independently accounted for, if they are not reproduced in local SN spectra. Also, the fact that in both cases we use the observed SN host galaxy itself as a template to decompose the extracted SN trace into a supernova and a galaxy component - case (b) in the above system of equations - illustrates what we were implying in Sect. 4 about the propagation of supernova "features" in the final SN spectrum through the inherent imperfections of the galaxy template removal approach.

\section{Conclusion}

We have presented the advantages of using a more elaborate spectral extraction algorithm for obtaining supernova spectra devoid of background contamination, and hence make possible a truly quantitative analysis of high- $z$ Type Ia supernova spectra. This will allow us in the future to set constraints on potential evolutionary effects that could affect SNe Ia as function of redshift. Not only are we able to extract the PSF-like components of an input spectrum, we can also restore the entire 2D spectrum and hence get a hold on systematic errors linked with the restoration process. We thus have a quantitative way of judging whether the extracted SN spectrum is optimal for subsequent analysis and whether we are not mistakenly "seeing" $\mathrm{SN}$-like features in a pure galaxy spectrum, as is sometimes the case in low $\mathrm{S} / \mathrm{N}$ high- $z \mathrm{SN}$ Ia spectra.

However, there are some caveats mainly associated to the complexity of specinholucy. Errors associated with the use of an inappropriate SSF for restoring the point-source channel or a mis-evaluation of the width of the spatial resolution kernel necessary for the point-source/extended background discrimination will strongly affect the restoration (see Sect. 3). Through restoration of the $2 \mathrm{D}$ background spectrum one is however able to produce diagnostic plots to critically evaluate the error in

\footnotetext{
${ }^{6}$ ESO-MIDAS is a registered trademark of the European Southern Observatory; see http://www . eso.org/projects/esomidas/
}

each case and repeat the restoration with different settings (see Fig. 5).

Note that a valuable by-product of this novel technique is the acquisition of a host galaxy spectrum with no contamination from the supernova flux! Galaxies at $z \sim 0.5$ are at most a few arcseconds in size and one can essentially obtain integrated spectra of high- $z$ SN host galaxies by centering a slit on both the SN and its host. This in turn makes it possible to study of properties of high- $z$ SN Ia host galaxies and compare them with integrated spectra of local SN Ia hosts (Sullivan et al. 2003; Williams et al. 2003; Ghallager \& Garnavich 2004).

Thus, specinholucy provides the high- $z$ supernova community with a reliable tool to obtain clean SN Ia spectra and in turn to derive accurate physical quantities associated with the ejecta. Two of the authors (Stéphane Blondin and Bruno Leibundgut) are part of the ESSENCE project, and Grégory Sainton is part of the SNLS collaboration. Both teams recognise the need for a quantitative analysis of their results in order to constrain potential systematic effects that could distort the cosmological results derived from observations of high- $z$ $\mathrm{SNe}$ Ia. We have shown specinholucy to be the ideal tool for this purpose. In fact, the VLT data taken for the ESSENCE project will be shown in their restored form via specinholucy in Matheson et al. (2005).

Acknowledgements. Many thanks to John Moustakas for providing the zero-point for the spectrum of NGC 6181 and to Mattia Vaccari for deriving the galaxy luminosity profile used in the simulation. We further would like to thank the anonymous referee for many detailed and useful comments on the original manuscript.

\section{References}

Avila, G., Rupprecht, G., \& Beckers, J. M. 1997, in Optical Telescopes of Today and Tomorrow, ed. A. L. Ardeberg, Proc. SPIE, 2871, 1135

Barris, B. J., Tonry, J. L., Blondin, S., et al. 2004, ApJ, 602, 571

de Vaucouleurs, G. 1959, Handbuch der Physik, 53, 275

Drenkhahn, G., \& Richtler, T. 1999, A\&A, 349, 877

Filippenko, A. V. 1997, ARA\&A, 35, 309

Freeman, K. C. 1970, ApJ, 160, 811

Fried, D. L. 1965, Opt. Soc. Am. J., 55, 1427

Fried, D. L. 1975, Radio Science, 10, 71

Ghallager, S., \& Garnavich, P. 2004, in preparation

Hillebrandt, W., \& Niemeyer, J. C. 2000, ARA\&A, 38, 191

Hook, R. N., \& Lucy, L. B. 1994, in The Restoration of HST Images and Spectra - II, 86

Kennicutt, R. C. 1992, ApJS, 79, 255

Kent, S. M. 1985, ApJS, 59, 115

Knop, R. A., Aldering, G., Amanullah, R., et al. 2003, ApJ, 598, 102

Leibundgut, B. 2001, ARA\&A, 39, 67

Lucy, L. B. 1974, AJ, 79, 745

Lucy, L. B. 1994, in The Restoration of HST Images and Spectra - II, 79

Lucy, L. B., \& Walsh, J. R. 2003, AJ, 125, 2266

Matheson, T., Blondin, S., Foley, R., et al. 2005, AJ, submitted

[arXiv: astro-ph/0411357]

Miknaitis, G., Tonry, J., Garnavich, P., et al. 2005, ApJ, submitted 
Nugent, P., Phillips, M., Baron, E., Branch, D., \& Hauschildt, P. 1995, ApJ, 455, L147

Pain, R., \& SNLS Collaboration. 2002, BAAS, 34, 1169

Patat, F. 2003, A\&A, 400, 1183

Patat, F., Benetti, S., Cappellaro, E., et al. 1996, MNRAS, 278, 111

Perlmutter, S., Aldering, G., Goldhaber, G., et al. 1999, ApJ, 517, 565

Phillips, M. M. 1993, ApJ, 413, L105

Ratnatunga, K. U., Griffiths, R. E., \& Ostrander, E. J. 1999, AJ, 118, 86

Richardson, W. H. 1972, Opt. Soc. Am. J., 62, 55

Riess, A. G., Filippenko, A. V., Challis, P., et al. 1998a, AJ, 116, 1009

Riess, A. G., Nugent, P., Filippenko, A. V., Kirshner, R. P., \& Perlmutter, S. 1998b, ApJ, 504, 935

Riess, A. G., Strolger, L., Tonry, J., et al. 2004, ApJ, 600, L163

Sainton, G. 2004, Ph.D. thesis, Université Lyon I, Nºrdre: 131-2004
Sandrock, S., Amestica, R., Duhoux, P., Navarrete, J., \& Sarazin, M. S. 2000, in Advanced Telescope and Instrumentation Control Software, ed. H. Lewis, Proc. SPIE, 4009, 338

Sarazin, M., \& Roddier, F. 1990, A\&A, 227, 294

Schmidt, B. P., Suntzeff, N. B., Phillips, M. M., et al. 1998, ApJ, 507, 46

Schroeder, D. J. 1987, Astronomical Optics (San Diego: Academic Press)

Sil'chenko, O. K., Zasov, A. V., Burenkov, A. N., \& Boulesteix, J. 1997, A\&AS, 121, 1

Sullivan, M., Ellis, R. S., Aldering, G., et al. 2003, MNRAS, 340, 1057

Tonry, J. L., Salvo, M., Blondin, S., Schmidt, B. P., \& Leibundgut, B. 2005 , in preparation

Tonry, J. L., Schmidt, B. P., Barris, B., et al. 2003, ApJ, 594, 1

Williams, B. F., Hogan, C. J., Barris, B., et al. 2003, AJ, 126, 2608 